\title{
Editorial
}

\section{Making Epidemiological Data Useful for Health Risk Assessment}

Risk assessment is a cross-disciplinary subject that is defined only by the problem to be solved and has no clear scientific domain boundaries. Procedures for health risk assessment are evolving continuously, and something better is always in need. Although epidemiological data have been playing important roles in health risk assessment, the need for innovation remains; therefore, it is desirable to make a concerted effort to explore their potential utility. In this special issue, a broad range of issues relevant to the use of epidemiological data for health risk assessment are addressed.

Appropriate human data from epidemiological studies, if available, provide the most direct information for health risk.

assessment because they avoid the need to extrapolate from animals to humans. The need to make epidemiological data more useful for risk assessment has been recognized in the past as underscored by a series of editorials and commentaries [1-5]. Epidemiological studies can be made more useful for health risk assessment by improving study designs, and/or methods of data analysis. For instance, Woodruff et al. [6] suggests that human data can be made more useful for risk assessment if some early biomarkers can be identified. They reviewed what was known about the relationship between chemical exposure, early biological effects (upstream events), and later overt effects (down stream events) through case studies of chemical-induced ill effects, and concluded that certain classes of upstream events can serve as useful biomarkers for identifying hazard, and risk prediction. With respect to data analysis, epidemiological data have traditionally been used to estimate risk to humans due to exposure to an agent [7-11]. Recently, epidemiological data have been used to characterize the lingering effects after cessation, or reduction of exposures by exploiting the dynamic nature of these data where each individual can be considered as an independent experiment with different exposure scenarios and follow up periods [12, 13]. The concept of effect lingering is closely related to that of cessation lag, the time required for risk to reduce to a certain level after cessation or reduction of exposure. The concept of cessation lag is useful for the analysis of economic benefits of an environmental regulation because the result is sensitive to the timing of the risk reductions and cannot be effectively addressed by the conventional dose-response procedures. Therefore, both risk estimation, and characterization of lingering and cessation effect are useful for environmental management, and regulatory decision making.

Scientific information used for health risk assessments usually comes from three sources: laboratory animal experiments, epidemiological studies, and in vivo/in vitro assays. The information from these three sources has been used for hazard identification, but its potential utility in risk assessment and decision making remains to be explored. Thus, how to use the information from these three sources more effectively, either separately, or jointly, is an area of great research interest. Finally, it is important to point out that some information from the rapidly emerging field of molecular biology, such as expression microarrays and/or proteomics, are observational data similar to those from epidemiological studies [14]. This is in contrast to the well controlled animal experiments for which confounding and selecting bias are eliminated through the use of properly designed experiments. For observational data, confounding factors, and selection bias are crucial factors that need to be carefully evaluated and eliminated. These issues, however, are not always properly addressed when such data are involved. Today, we are standing at an opportune point in time, able to take advantage of both the emerging observational and laboratory controlled data, and to use them to advance health risk assessment. It should be acknowledged, however, that many emerging issues such as those involving the use of genomic data for risk assessment have not been fully explored here, although they have been discussed in some of the papers. These issues remain open and require further research.

*The ideas and viewpoints in this commentary are those of the author and do not necessarily represent the positions or policies of the USEPA

\section{REFERENCES}

[1] Shore R. Editorial: epidemiologic data in risk assessment-imperfect but valuable. Am J Public Health 1995; 85(4): 474-5.

[2] Hertz-Picciotto I. Epidemiology and quantitative risk assessment: A bridge from science to policy. Am J Public Health 1995; 85(4): 484-91.

[3] Wartenberg D, Simon R. Comment: Integrating epidemiologic data into risk assessment. Am J Public Health 1995; 85(4): 491.

[4] Samet JM, Schnatter R, Gibb H. Invited commentary: epidemiology and risk assessment. Am J Epidemiol 1998; 148: 929-36.

[5] Goldman L. Epidemiology in the regulatory arena. Am J Epidemiol 2005; 154(S12): 18-26.

[6] Woodruff T, Zeise L, Axelrad D, et al. Moving upstream: Evaluating adverse upstream endpoints for improved risk assessment and decision-making. Environ Health Perspect 2008; 116: 1657-75.

[7] Stayner L, Smith R, Thun M, et al. A dose-response analysis and quantitative assessment of lung cancer risk and occupational cadmium exposure. Ann Epidemiol 1992; 2(3): 177-94.

[8] Crump K. Risk of benzene-induced leukemia predicted from the pliofilm cohort. Environ Health Perspect 1996; 104(S6): 1437-41.

[9] Bender R. Quantitative risk assessment in epidemiological studies investigating threshold effects. Biometrical J 1999; 41(3): 305-19.

[10] Meijers J, Swaen G, Schreiber G, Sturmans F. Occupational epidemiological studies in risk assessment and their relation to animal experimental data. Regul Toxicol Pharmacil 1992; 16(3): 215-22. 
[11] Axelrad D, Bellinger D, Ryan L, Woodruff T. Dose-response relationship of prenatal mercury exposure and IQ: an integrative analysis of epidemiologic data. Environ Health Perspect 2007; 115(4): 609-15.

[12] Chen C, Gibb H. Procedures for calculating cessation lag. Regul Toxicol Pharmacol 2003; 38: 157-65.

[13] Chen C. Lingering effect: epidemiological information useful for risk assessment. Regul Toxicol Pharmacol 2008; 52: 242-7.

[14] Potter J. Epidemiology, cancer genetics and microarrays: making correct inferences, using appropriate designs. Trends Genet 2003; 19(12): 690-5.

\section{Chao W. Chen}

(Guest Editor)

National Center of Environmental Assessment

U.S. Environmental Protection Agency

Washington DC

USA

E-mail: Chen.Chao@epamail.epa.gov

(C) Chao W. Chen; Licensee Bentham Open.

This is an open access article licensed under the terms of the Creative Commons Attribution Non-Commercial License (http://creativecommons.org/licenses/by$\mathrm{nc} / 3.0 /$ ) which permits unrestricted, non-commercial use, distribution and reproduction in any medium, provided the work is properly cited. 